\title{
Beat wave injection of electrons into plasma waves using two interfering laser pulses
}

\author{
G. Fubiani, ${ }^{*}$ E. Esarey, C.B. Schroeder, and W.P. Leemans \\ Center for Beam Physics, Ernest Orlando Lawrence Berkeley National Laboratory, \\ University of California, Berkeley, CA 94720
}

(Dated: March 4, 2004)

\begin{abstract}
An electron injector concept that uses a single injection laser pulse colliding with a pump laser pulse in a plasma is analyzed. The pump pulse generates a large amplitude laser wakefield (plasma wave). The counterpropagating injection pulse collides with the pump laser pulse to generate a beat wave with a slow phase velocity. The ponderomotive force of the slow beat wave is responsible for injecting plasma electrons into the wakefield near the back of the pump pulse. Test particle simulations indicate that significant amounts of charge can be trapped and accelerated $(\sim 10 \mathrm{pC})$. For higher charge, beam loading limits the validity of the simulations. The accelerated bunches are ultrashort $(\sim 1 \mathrm{fs}$ ) with good beam quality (relative energy spread of a few percent at a mean energy of $\sim 10 \mathrm{MeV}$ and a normalized rms emittance on the order $0.4 \mathrm{~mm} . \mathrm{mrad}$ ). The effects of interaction angle and polarization are also explored, e.g., efficient trapping can occur for near-collinear geometries. Beat wave injection using a single injection pulse has the advantages of simplicity, ease of experimental implementation, and requires modest laser intensity $I \simeq 8.8 \times$ $10^{17} \mathrm{~W} / \mathrm{cm}^{2}$.
\end{abstract}

PACS numbers: 52.38.Kd, 52.38.-r

\footnotetext{
*Also at University of Paris XI (Orsay), France; Electronic address: GJFubiani@lbl.gov
} 


\section{INTRODUCTION}

Plasma-based accelerators [1] are capable of producing compact and high energy electron sources in much shorter distances than conventional accelerators due to the large longitudinal electric fields that can be excited without the limitation of breakdown as in RF structures. In a plasma, the accelerating fields of a plasma wave are on the order of the cold, nonrelativistic wavebreaking field $E_{0}=m_{e} c \omega_{p} / e$, or $E_{0}[\mathrm{~V} / \mathrm{m}] \simeq 96\left(n_{0}\left[\mathrm{~cm}^{-3}\right]\right)^{1 / 2}$, where $\omega_{p}=\left(4 \pi n_{0} e^{2} / m_{e}\right)^{1 / 2}$ is the plasma frequency, $n_{0}$ is the plasma density, $c$ is the speed of light, $m_{e}$ the electron mass, and $e$ the electron charge. The wavelength of the accelerating field is the plasma wavelength $\lambda_{p}=2 \pi c / \omega_{p}$, or $\lambda_{p}[\mathrm{~m}] \simeq 3.3 \times 10^{4}\left(n_{0}\left[\mathrm{~cm}^{-3}\right]\right)^{-1 / 2}$. For example, a laser wakefield accelerator (LWFA) [1] in the standard regime, in which the laser pulse length $L$ is matched to the plasma wavelength, $L \simeq \lambda_{p}$, typically has a density on the order of $n_{0} \simeq 10^{18} \mathrm{~cm}^{-3}$ for a 100 fs pulse, which gives $E_{0} \simeq 100 \mathrm{GV} / \mathrm{m}$ and $\lambda_{p} \simeq 30 \mu \mathrm{m}$. If a mono-energetic electron bunch is injected into a wakefield such that it is accelerated while maintaining a small energy spread, then it is necessary for the bunch to occupy a small fraction of the wakefield period, on the order of a few femtoseconds, which requires femtosecond accuracy in the injection process. To meet these requirements, a variety of laser injection methods have been proposed [2-7].

Perhaps the most basic and simplest form of a laser-plasma injector is the self-modulated LWFA $[1,8]$, in which a single laser pulse, propagating in a relatively high density plasma (such that $L>\lambda_{p}$ and the laser pulse power exceeds the critical power for relativistic focusing), results in self-trapping and generation of a sub-ps electron bunch, however, with a large energy spread. Typically the self-trapped bunch is of high charge (up to $10 \mathrm{nC}$ ), with an energy distribution that can be modeled as a Boltzmann distribution with temperature in the few MeV range [9-14]. One possible mechanism for self-trapping is direct wavebreaking of the plasma wakefield [15]. Since the phase velocity of the wakefield is near the speed of light, it is difficult to trap the background fluid electrons, which are undergoing the fluid oscillation that sustains the wakefield. Wavebreaking typically occurs at high wakefield amplitudes, e.g., amplitudes greater than the wavebreaking field, which for a cold one-dimensional (1D) plasma wave is $E_{\mathrm{WB}}=\left[2\left(\gamma_{p}-1\right)\right]^{1 / 2} E_{0} \gg E_{0}$, where $v_{p}=c \beta_{p}=c\left(1-\gamma_{p}^{-2}\right)^{1 / 2}$ is the phase velocity of the plasma wave. Alternatively, self-trapping and acceleration can result from the coupling of Raman backscatter and Raman sidescatter to the wakefield [16]. When electrons 
become trapped in the fast wakefield, they become accelerated to high energies as they rotate up in momentum inside the separatrix of the wakefield. In the self-modulated regime, a large energy spread for the trapped electrons results because (i) some fraction of the background electrons are continually being swept up and trapped in the wakefield as the laser pulse propagates into fresh plasma, and (ii) typically the self-guided propagation distance of the laser pulse is much greater than the detuning length for trapped electrons. This implies that deeply trapped electrons will circulate many revolutions within the separatrix, again resulting in a large energy spread.

For many applications, a small energy spread is desired. This can be achieved by using a standard LWFA, in which the wakefield is produced in a controlled manner at an amplitude below the wavebreaking or self-trapping threshold. In principle, if a small energy spread electron bunch of duration small compared to $\lambda_{p}$ is injected into the wakefield at the proper phase, then the bunch can be accelerated while maintaining a small energy spread. Umstadter et al. [2] first proposed using an additional laser pulse to inject background plasma electrons into the wave for acceleration to high energies. To generate ultrashort electron bunches with low energy spreads, the original laser injection method proposed by Umstadter et al. [2] (referred to as the LILAC scheme) utilizes two laser pulses which propagate perpendicular to one another. The first pulse (pump pulse) generates the wakefield via the standard LWFA mechanism, and the second pulse (injection pulse) intersects the wakefield some distance behind the pump pulse. The ponderomotive force $\boldsymbol{F} \simeq-\left(m_{e} c^{2} / \gamma\right) \nabla a^{2} / 2$ of the injection pulse can accelerate a fraction of the plasma electrons such that they become trapped in the wakefield. Here $\gamma$ is the relativistic Lorentz factor of the electrons and $a^{2} \simeq 3.6 \times 10^{-19}(\lambda[\mu \mathrm{m}])^{2} I\left[\mathrm{~W} / \mathrm{cm}^{2}\right]$ for a circularly polarized laser field, with $\lambda$ the laser wavelength and $I$ the laser intensity. Specifically, the axial (direction of propagation of the pump pulse along the $z$-axis) ponderomotive force of the injection pulse (propagating along the $x$-axis) scales as

$$
F_{z, \text { pond }}=-\left(m_{e} c^{2} / \gamma\right)(\partial / \partial z) a_{1}^{2} / 2 \sim\left(m_{e} c^{2} / \gamma\right) a_{1}^{2} / r_{1}
$$

where $a_{1}^{2}$ and $r_{1}$ are the normalized intensity and spot size of the injection pulse, respectively. A simple estimate for the change of momentum that an electron will experience due to the ponderomotive force of the injection pulse is $\Delta p_{z} \simeq \tau_{1} F_{z \text {,pond }} \sim\left(m c^{2} / \gamma\right) a_{1}^{2} \tau_{1} / r_{1}$, where $\tau_{1}$ is the injection pulse duration. It is possible for $\Delta p_{z}$ to be sufficiently large that electrons are 
injected into the separatrix of the wakefield such that they become trapped and accelerated to high energies. To inject into a single plasma wave bucket, it is necessary for both the injection pulse spot size and pulse length to be small compared to the plasma wavelength, i.e., $r_{1}^{2} \ll \lambda_{p}^{2}$ and $c^{2} \tau_{1}^{2} \ll \lambda_{p}^{2}$. Simulations [2], which were performed for ultrashort pulses at high densities $\left(\lambda_{p} / \lambda=10\right.$ and $\left.E_{z} / E_{0}=0.7\right)$, indicated the production of a $10 \mathrm{fs}, 21 \mathrm{MeV}$ electron bunch with a $6 \%$ energy spread. However, high intensities $\left(I>10^{18} \mathrm{~W} / \mathrm{cm}^{2}\right)$ are required in both the pump and injection pulses $\left(a_{0} \simeq a_{1} \simeq 2\right)$. It is important to note that in the work of Umstadter et al. [2], the pump pulse and the injection pulse do not overlap (in space and time) and a laser beat wave is not generated, as is discussed below.

Hemker et al. [4] also studied the LILAC injection scheme using 2D particle-in-cell simulations. They found that the wake generated by the transverse propagating injection pulse can play an important role in the trapping process and even exceed the amount of trapping produced by the ponderomotive force of the injection pulse alone. In addition, they varied the delay between the pump and injection pulses and found that the trapping can be enhanced when the two pulses overlap. However, the electric field polarizations of the two pulses were orthogonal in these simulations, i.e., no laser beat wave was generated when the two pulses overlapped.

Esarey et al. $[3,6]$ proposed and analyzed a colliding pulse injection (CPI) concept that uses three short laser pulses: an intense $\left(a_{0}^{2} \simeq 1\right.$ ) pump pulse (denoted by subscript 0 ) for plasma wave generation, a forward going injection pulse (subscript 1), and a backward going injection pulse (subscript 2). CPI is intrinsically different from the method of ponderomotive injection discussed above in that both the source and form of the ponderomotive force, responsible for injection, differs in these two methods. In ponderomotive injection, injection is the result of the ponderomotive force associated with the envelope (time-averaged intensity profile) of a single pulse. In CPI, injection is the result of the ponderomotive force associated with the slow beat wave of two intersecting pulses.

In CPI, the pump pulse generates a plasma wave with phase velocity near the speed of light $\left(v_{p 0} \simeq c\right)$. The forward injection pulse travels at a fixed distance behind the pump pulse, which determines the position (i.e., phase) of the injected electrons. The injection pulses are orthogonally polarized to the pump laser pulse, such that the pump pulse and backward going injection pulse do not beat. When the injection pulses collide some distance behind the pump, they generate a slow ponderomotive beat wave of the form $a_{1} a_{2} \cos (\Delta k z-\Delta \omega t)$ 
(here $\Delta k=k_{1}-k_{2} \simeq 2 k_{0}$ ) with a phase velocity $v_{p b} \simeq|\Delta \omega| / 2 k_{0} \ll c$, where the frequency, wavenumber, and normalized intensity of the pulses are denoted by $\omega_{i}, k_{i}$, and $a_{i}(i=0,1,2)$, respectively. Furthermore, it is assumed that $k_{1} \simeq k_{0}, k_{2} \simeq-k_{0}$, and $\omega_{1}-\omega_{2}=\Delta \omega \gg \omega_{p}$. The axial force associated with this beat wave scales as

$$
F_{z, \text { beat }}=-\left(m_{e} c^{2} / \gamma\right)(\partial / \partial z) a_{1} a_{2} \cos \left(2 k_{0} z-\Delta \omega t\right) \sim\left(m_{e} c^{2} / \gamma\right) 2 k_{0} a_{1} a_{2}
$$

During the time in which the two injection pulses overlap, a two-stage acceleration process can occur, i.e., the slow beat traps and heats background plasma electrons which, as a result of shifts in their momentum and phase, can be injected into the fast wakefield for acceleration to high energies.

The ratio of the axial force of the CPI beat wave to that of a single pulse in the ponderomotive injection scheme scales as

$$
\frac{F_{z, \text { beat }}}{F_{z, \text { pond }}} \sim \frac{2 k_{0} a_{1} a_{2}}{a_{p}^{2} / r_{p}},
$$

where the subscript $p$ refers to the single ponderomotive injection pulse and the contribution of the relativistic Lorentz factor $\gamma$ (which is different for the two cases) is neglected. For comparable injection pulse intensities $\left(a_{1} \simeq a_{2} \simeq a_{p}\right)$, the ratio scales as $2 k_{0} r_{p} \gg 1$, i.e., the axial force of the beat wave is much greater than the ponderomotive force of a single pulse. Consequently, CPI using beat waves is much more effective for electron injection than relying on the ponderomotive force of the injection pulse alone. CPI can result in electron injection at relatively low intensities $\left(a_{1} \sim a_{2} \sim 0.2\right)$, as well as at relatively low densities $\left(\lambda_{p} / \lambda \sim 100\right)$, thus allowing for high single-stage energy gains. Furthermore, the CPI concept offers detailed control of the injection process: the injection phase can be controlled via the position of the forward injection pulse, the beat phase velocity via $\Delta \omega$, the injection energy via the pulse amplitudes, and the injection time (number of trapped electrons) via the backward pulse duration.

In this article, a simplified configuration of the CPI concept is proposed and analyzed that uses only two laser pulses with parallel polarizations: an intense pump pulse for wakefield generation and a single counterpropagating (or propagating at a finite angle) injection pulse. Injection is the result of the laser beat wave produced when the backward injection pulse collides with the trailing portion of the pump pulse. This configuration has the advantages of being easier to implement in comparison to the three-pulse CPI scheme, and of requiring 
less intensity in the injection pulse compared to the ponderomotive injection scheme, since injection is the result of the laser beat wave as opposed to the ponderomotive force of a single injection pulse.

In the following, analytical models and test particle simulations are used to describe the basic characteristics of the two-pulse CPI concept, such as the threshold for injection and the trapped bunch quality. Test particle simulations are carried out in three dimensions (3D) in which the fields of the laser pulses and their wakes are described analytically via linear theory. For high laser intensities $\left(a^{2}>1\right)$, this model becomes inaccurate. To describe the nonlinear regime in 3D, as well as other nonlinear effects such as beam loading, requires self-consistent simulations such as can be done with particle-in-cell codes, which is beyond the scope of this paper. Also explored are the effects of interaction angle and polarization on the injection process. These results are directly relevant to laser injection experiments being pursued at Lawrence Berkeley National Laboratory (LBNL) [17] and elsewhere.

\section{TWO-PULSE CPI: FIELDS}

This section describes the fields used in the two-pulse CPI simulations discussed below. The laser fields of the pump $(i=0)$ and injection $(i=1)$ laser pulses are described by the normalized vector potentials $\boldsymbol{a}_{i}=e \boldsymbol{A}_{i} / m_{e} c^{2}$. Using the paraxial wave equation with a linear plasma response, the transverse laser fields (linearly polarized in the $x$-direction and propagating along the $z$-axis) are given by [18]

$$
a_{x i}\left(r, \zeta_{i}\right)=\hat{a}_{i}\left(r, \zeta_{i}\right) \cos \psi_{i}
$$

with

$$
\hat{a}_{i}\left(r, \zeta_{i}\right)=a_{i}\left(r_{i} / r_{s i}\right) \exp \left(-r^{2} / r_{s i}^{2}\right) \sin \left(\pi \zeta_{i} / L_{i}\right)
$$

for $-L_{i}<\zeta_{i}<0$ and zero otherwise, where $\zeta_{0}=z-\beta_{g 0} c t$ (forward comoving coordinate), $\zeta_{1}=z+\beta_{g 1} c t$ (backward comoving coordinate), $\beta_{g i}=\eta_{i}$ is the linear group velocity, $\beta_{\phi i}=\eta_{i}^{-1}$ is the linear phase velocity, $\eta_{i}=\sqrt{1-\omega_{p}^{2} / \omega_{i}^{2}-4 /\left(k_{i} r_{i}\right)^{2}}$ is the plasma index of refraction, $\psi_{i}=k_{i}\left(z-\beta_{\phi i} c t\right)+\alpha_{i} r^{2} / r_{s i}^{2}+\alpha_{i}-\tan ^{-1} \alpha_{i}$ is the phase, $k_{i}=\omega_{i} /\left(\beta_{\phi i} c\right)$ is the wavenumber, $\omega_{i}$ is the frequency in vacuum, $r_{s i}(z)=r_{i} \sqrt{1+\alpha_{i}(z)}$ is the spot size, $r_{i}$ is the spot size at waist (here chosen to be $\left.z=Z_{f_{i}}\right), \alpha_{i}(z)=\left(z-Z_{f_{i}}\right)^{2} / Z_{R_{i}}^{2}, Z_{R_{i}}=k_{i} \eta_{i} r_{i}^{2} / 2$ is the Rayleigh length, $L_{i}$ is the pulse length, and a constant has been omitted in the definition of $\psi_{i}$ that 
represents the initial position and phase of the laser pulse. The axial component of the laser field is specified via $\nabla \cdot \boldsymbol{a}_{i}=0$. Keeping only the leading order contributions gives

$$
\begin{aligned}
a_{z i}\left(r, \zeta_{i}\right) & =-\int_{0}^{\zeta_{i}} d \zeta_{i}^{\prime} \partial a_{x i}\left(r, \zeta_{i}^{\prime}\right) / \partial x \\
& \simeq 2 x\left[\hat{a}_{i}\left(r, \zeta_{i}\right) /\left(k_{i} r_{s i}^{2}\right)\right]\left(\sin \psi_{i}-\alpha_{i} \cos \psi_{i}\right) .
\end{aligned}
$$

For simplicity, the notation $\left\langle a_{i}^{2}\right\rangle$ is introduced to denote the time-averaged peak intensity of the laser pulse. For a linearly polarized laser pulse of the form $a_{i} \cos \psi_{i} e_{x},\left\langle a_{i}^{2}\right\rangle=a_{i}^{2} / 2$. For a circularly polarized laser pulse of the form $a_{i}\left(\cos \psi_{i} e_{x}+\sin \psi_{i} e_{y}\right),\left\langle a_{i}^{2}\right\rangle=a_{i}^{2}$. Comparisons between linear and circular polarization will be done for equal values of the time-averaged peak intensity $\left\langle a_{i}^{2}\right\rangle$. The weakly relativistic limit, sometimes referred to as the linear regime, corresponds to $\left\langle a_{i}^{2}\right\rangle \ll 1$.

Included in the simulations presented in Sec. IV are the wakefields generated by both the pump and injection laser pulses. In the linear $\left(\left\langle a_{i}^{2}\right\rangle \ll 1\right)$ three-dimensional (3D) regime, wakefield generation can be examined using the cold fluid equations. In particular for linear polarization, the normalized electrostatic potential of the wakefield $\phi_{i}=e \Phi_{i} / m_{e} c^{2}$ is given by $[1]$

$$
\left(\partial^{2} / \partial \zeta_{i}^{2}+k_{p}^{2}\right) \phi_{i} \simeq k_{p}^{2} \hat{a}_{i}^{2} / 4
$$

where $k_{p}=\omega_{p} / c$ and a time-averaging has been performed over the fast laser oscillation (laser frequency), i.e., $\left\langle\hat{a}_{i}^{2} \cos ^{2} \psi_{i}\right\rangle=\hat{a}_{i}^{2} / 2$. The solution to Eq. (7) is

$$
\phi_{i}\left(r, \zeta_{i}\right)=k_{p} \int_{0}^{\zeta_{i}} d \zeta_{i}^{\prime} \sin k_{p}\left(\zeta_{i}-\zeta_{i}^{\prime}\right) \hat{a}_{i}^{2}\left(r, \zeta_{i}^{\prime}\right) / 4
$$

Specifically, Eq. (7) yields the potential generated inside the pulse $\left(-L_{i}<\zeta_{i}<0\right)$

$$
\phi_{i}=\frac{a_{i}^{2}}{8} \frac{r_{i}^{2}}{r_{s i}^{2}} e^{-2 r^{2} / r_{s i}^{2}}\left[1+\frac{\left(4 \pi^{2} / k_{p}^{2} L_{i}^{2}\right) \cos \left(k_{p} \zeta_{i}\right)-\cos \left(2 \pi \zeta_{i} / L_{i}\right)}{\left(1-4 \pi^{2} / k_{p}^{2} L_{i}^{2}\right)}\right],
$$

and behind the pulse $\left(\zeta_{i}<-L_{i}\right)$

$$
\phi_{i}=\frac{a_{i}^{2}}{4} \frac{r_{i}^{2}}{r_{s i}^{2}} e^{-2 r^{2} / r_{s i}^{2}}\left(\frac{4 \pi^{2}}{k_{p}^{2} L_{i}^{2}}\right) \frac{\sin \left[k_{p}\left(\zeta_{i}+L_{i} / 2\right)\right] \sin \left(k_{p} L_{i} / 2\right)}{\left(1-4 \pi^{2} / k_{p}^{2} L_{i}^{2}\right)} .
$$

For the resonant case $L=\lambda_{p}$, which corresponds to maximum wakefield generation,

$$
\phi_{i}=\frac{a_{i}^{2}}{8} \frac{r_{i}^{2}}{r_{s i}^{2}} e^{-2 r^{2} / r_{s i}^{2}}\left[1-\cos \left(k_{p} \zeta_{i}\right)-\left(k_{p} \zeta_{i} / 2\right) \sin \left(k_{p} \zeta_{i}\right)\right]
$$

and

$$
\phi_{i}=\frac{\pi a_{i}^{2}}{8} \frac{r_{i}^{2}}{r_{s i}^{2}} e^{-2 r^{2} / r_{s i}^{2}} \sin \left(k_{p} \zeta_{i}\right)
$$


within and behind the pulse, respectively.

During the collision (overlap) of the two laser pulses, a beat wave space charge potential $\phi_{b}$ will be driven by the slow ponderomotive beat wave, i.e.,

$$
\left(\partial^{2} / \partial c t^{2}+k_{p}^{2}\right) \phi_{b} \simeq k_{p}^{2}\left\langle a_{x 0} a_{x 1}\right\rangle
$$

where $\left\langle a_{x 0} a_{x 1}\right\rangle=\left(\hat{a}_{0} \hat{a}_{1} / 2\right) \cos \psi_{b}, \psi_{b}=\Delta k\left(z-\beta_{b} c t\right)$ is the beat wave phase, $c \beta_{b}=\Delta \omega / \Delta k$ is the beat wave phase velocity, $\Delta \omega=\omega_{0}-\omega_{1}$, and $\Delta k=k_{0}-k_{2} \simeq 2 k_{0}$ assuming $\Delta \omega^{2} \ll \omega_{i}^{2}$ and a counterpropagating geometry. As an example, analytical solutions for $\phi_{b}$ can be found in the linear limit for the case of square pulse profiles, without diffraction and equal frequencies $\Delta \omega=0$. In this case, during the overlap,

$$
\phi_{b}=\left(a_{0} a_{1} / 4\right)\left[1-\cos \omega_{p}\left(t-t_{\mathrm{on}}\right)\right] \cos \psi_{b},
$$

where $t_{\text {on }}(z)$ is the onset of overlap of the colliding pulses at fixed $z$. Associated with $\phi_{b}$ is a density perturbation $\delta n_{b}=n_{0} k_{p}^{-2} \nabla^{2} \phi_{b}$, i.e., $\delta n_{b} / n_{0} \simeq-\left(2 k_{0} / k_{p}\right)^{2} \phi_{b}$. Strictly speaking, the linear solution given by Eq. (14) is only valid if $\left|\delta n_{b} / n_{0}\right| \ll 1$, or $\left(k_{0} / k_{p}\right)^{2} a_{0} a_{1} \ll 1$, which is easily violated even for modest values of $a_{0} a_{1}$ since $\left(k_{0} / k_{p}\right)^{2} \gg 1$. However, the relation $\nabla^{2} \phi_{b}=k_{p}^{2} \delta n_{b} / n_{0}$ holds in the nonlinear limit and hence the scaling $\left|\phi_{b}\right| \sim\left(k_{p} / 2 k_{0}\right)^{2}\left|\delta n_{b} / n_{0}\right|$ holds even for large values of $\delta n_{b} / n_{0}$, assuming $\nabla^{2} \phi_{b} \sim 4 k_{0}^{2} \phi_{b}$. In particular, as long as $\left|\delta n_{b} / n_{0}\right| \ll\left(2 k_{0} / k_{p}\right)^{2}\left(a_{0} a_{1}\right)$ [e.g., $\left(2 k_{0} / k_{p}\right)^{2}\left(a_{0} a_{1}\right) \sim 10^{3}$ in the simulations presented below], then $\left|\nabla \phi_{b}\right| \ll\left|\nabla a_{0} a_{1} / 2\right|$ (i.e., $\left.\left|\phi_{b}\right| \ll a_{0} a_{1}\right)$ and the effects of the space charge potential of the beat wave $\phi_{b}$ can be neglected in comparison to the ponderomotive potential of the beat wave $a_{0} a_{1}$. Hence, in the following test particle simulations, $\phi_{b}$ is neglected.

\section{PHASE SPACE ANALYSIS}

To gain a qualitative understanding of the basic process, a heuristic theory of injection and trapping is presented. Specifically, an approximate expression for the injection threshold can be obtained by considering the motion of an electron in the wakefield and the beat wave individually, and by using an island overlap criteria [19]. Recall that the beat wave leads to formation of phase space buckets (separatrices) of width $2 \pi / \Delta k \simeq \lambda_{0} / 2$, which are much shorter than those of the wakefield $\left(\lambda_{p}\right)$, thus allowing for a separation of spatial scales. In the following analytical treatment, electron motion will be described using a Hamiltonian 
approach in the limit of a broad laser pulse $\left(r_{0} k_{p} \gg 1\right.$ and neglecting diffraction effects)

and assuming $\omega_{p}^{2} / \omega_{i}^{2} \ll 1$ (such that the group and phase velocities are approximately $c$ ). Furthermore, circular polarization will be assumed, $\mathbf{a}=\sum_{i} \hat{a}_{i}\left(\cos \psi_{i} \mathbf{e}_{x}+\sin \psi_{i} \mathbf{e}_{y}\right)$, such that $a^{2}=\hat{a}_{0}^{2}+\hat{a}_{1}^{2}+2 \hat{a}_{0} \hat{a}_{1} \cos \psi_{b}$ is independent of the fast laser phase $\psi_{i}$ and only a function of the beat phase $\psi_{b}=\psi_{0}-\psi_{1} \simeq 2 k_{0} z-\Delta \omega t$.

\section{A. Plasma wave}

In the absence of the beat wave $\left(a_{1}=0\right)$, the nonlinear motion of an electron in a plasma wave with relativistic phase velocity is described by the Lorentz equation, which in the 1D limit can be written in the following form [7]

$$
\begin{aligned}
\frac{d \psi}{d \omega_{p} t} & =\frac{\partial H}{\partial u_{z}}=\frac{u_{z}}{\sqrt{\gamma_{\perp}^{2}(\psi)+u_{z}^{2}}}-\beta_{\phi}, \\
\frac{d u_{z}}{d \omega_{p} t} & =-\frac{\partial H}{\partial \psi}=\frac{\partial \phi}{\partial \psi}-\frac{1}{2 \sqrt{\gamma_{\perp}^{2}(\psi)+u_{z}^{2}}} \frac{\partial \gamma_{\perp}^{2}}{\partial \psi},
\end{aligned}
$$

where $\boldsymbol{u}=\boldsymbol{p} / m_{e} c$ is the normalized electron momentum, $\gamma_{\perp}=\left(1+\hat{a}^{2}\right)^{1 / 2}, \psi=k_{p}\left(z-v_{\phi} t\right)$ is phase of the plasma wave, $v_{\phi}=c \beta_{\phi}=c\left(1-1 / \gamma_{\phi}^{2}\right)^{1 / 2}$ is the phase velocity of the plasma wave (approximately equal to the group velocity of the drive laser pulse), and

$$
H\left(u_{z}, \psi\right)=\sqrt{\gamma_{\perp}^{2}(\psi)+u_{z}^{2}}-\beta_{\phi} u_{z}-\phi(\psi) \text {. }
$$

is the Hamiltonian. Here, $\phi(\psi)$ is the laser-driven plasma wave (wakefield) potential given by Eq. (8) and the subscript 0, denoting the pump laser pulse, has been omitted. In the above equations, the identity $\mathbf{u}_{\perp}=\mathbf{a}$ has been used, which is exact in $1 \mathrm{D}$. Note that the Hamiltonian is time independent (a function of only $\psi$ ) and, therefore, is constant along any orbit.

The normalized axial momentum of an electron on an orbit (specified by the value of $H_{c}$ ) in the plasma wave is found from Eq. (17) by setting $H=H_{c}$, where $H_{c}$ is a constant, i.e.,

$$
u_{z}(\psi)=\beta_{\phi} \gamma_{\phi}^{2}\left(H_{c}+\phi\right) \pm \gamma_{\phi} \sqrt{\gamma_{\phi}^{2}\left(H_{c}+\phi\right)^{2}-\gamma_{\perp}^{2}}
$$

For example, assuming the plasma is initially cold (i.e., $u_{z}=0$ in front of the laser pulse where $a^{2}=\phi=0$ ), the background electron fluid motion in the plasma wave is defined by the orbit $H_{c}=1$. 
The Hamiltonian $H\left(u_{z}, \psi\right)$ exhibits fixed points $\left(d u_{z} / d t=d \psi / d t=0\right)$ that are stable ("O" points) at $u_{z}=\gamma_{\perp}\left(\psi_{\mathrm{o}}\right) \gamma_{\phi} \beta_{\phi}, \psi_{\mathrm{o}} \simeq-1.47-0.60 k_{p} L+0.02 k_{p}^{2} L^{2}$ inside the drive pulse and $\psi_{\mathrm{o}}=-\pi / 2-k_{p} L / 2$ modulo $2 \pi$ outside. Unstable fixed points ("X" points) lie at $u_{z}=\gamma_{\phi} \beta_{\phi}$ and $\psi_{\mathrm{x}}=-3 \pi / 2-k_{p} L / 2$ modulo $2 \pi$. The boundary between trapped and untrapped orbits defines the separatrix orbit, which is specified by $H_{c}=H\left(\gamma_{\phi} \beta_{\phi}, \psi_{\mathrm{x}}\right)$ and crosses the X-point.

Figure 1(a) shows wakefield $\phi$ (solid line), drive laser pulse envelope $\hat{a}^{2}$ (dashed line), and longitudinal electric field $E_{z}=-\partial_{z} \phi$ (dot-dashed line) for the parameters $L_{0}=\lambda_{p}$ and $\left\langle a_{0}^{2}\right\rangle=0.5$ as obtained from Eqs. (11)-(12). The corresponding phase space orbits are plotted in Fig. 1(b) as obtained from Eq. (18). Shown are the cold fluid orbit, separatrix between trapped and untrapped orbits, and the trapped and focused (2D) separatrix.

Behind the drive laser pulse, the width of the separatrix is $\Delta \psi=2 \pi$, however, only half this region is accelerating (the left half for the case of the laser pulse propagating to the right). The width of the accelerating region of the wakefield is $\Delta \psi=\pi$ and extends from the O-point to the X-point. When two-dimensional (2D) effects are taken into consideration (specifically, the focusing and defocusing regions associated with the transverse electric field of the plasma wave), there exists only a region of width $\Delta \psi=\pi / 2$ that is both accelerating and focusing (extending from the O-point to half the distance to the X-point). Hence, the "2D separatrix", defining the region of trapped orbits that are both accelerating and focusing, is given by $H_{c}=H\left(\gamma_{\phi} \beta_{\phi},-\pi-k_{p} L / 2\right.$ modulo $\left.2 \pi\right) \equiv H_{f}$. For the case of a single injection pulse colliding with the pump pulse, trapping will occur within the first 2D separatrix, which typically extends from the O-point within the pump laser pulse to roughly half the distance to the first X-point immediately behind the pump pulse. This region of trapped orbits that are in the accelerating and focusing region of the wakefield are characterized by values of the Hamiltonian in the range $H_{f}=H\left(\gamma_{\phi} \beta_{\phi},-\pi-k_{p} L / 2\right.$ modulo $\left.2 \pi\right) \leq H \leq H_{\mathrm{o}}=H\left(\gamma_{\phi} \beta_{\phi}, \psi_{\mathrm{o}}\right)$.

In the limit $\gamma_{\phi}^{2}\left(H_{c}+\phi\right)^{2} \gg \gamma_{\perp}^{2}$, Eq. (18) can be expanded to yield $u_{z}=2 \gamma_{\phi}^{2}\left(H_{c}+\phi\right)$ and $u_{z}=\gamma_{\perp}^{2} /\left[2\left(H_{c}+\phi\right)\right]-\left(H_{c}+\phi\right) / 2$ for the plus and minus portions of Eq. (18), respectively, assuming $\gamma_{\phi}^{2} \gg 1$. These expressions are useful for evaluating $u_{z}(\psi)$ on the separatrix for values of $\psi$ in the vicinity of the O-points. 


\section{B. Ponderomotive beat wave}

The motion of the electron in the beat wave alone $(\phi=0)$ is described by the beat wave Hamiltonian $[3,6,7]$

$$
H_{b}\left(u_{z}, \psi_{b}\right)=\sqrt{\gamma_{\perp b}^{2}\left(\psi_{b}\right)+u_{z}^{2}}-\beta_{b} u_{z}-\phi_{b}\left(\psi_{b}\right)
$$

where $\gamma_{\perp b}^{2}\left(\psi_{b}\right) \simeq 1+\hat{a}_{0}^{2}+2 \hat{a}_{0} \hat{a}_{1} \cos \psi_{b}\left(a_{1}^{2} \ll a_{0}^{2}\right.$ has been assumed $), \psi_{b}=\left(k_{0}-k_{1}\right)\left(z-\beta_{b} c t\right)$ is the beat wave phase (note $k_{1}<0$ for the backward pulse), and $c \beta_{b}=\Delta \omega /\left(k_{0}-k_{1}\right)$ is the beat wave phase velocity $\left(\Delta \omega=\omega_{0}-\omega_{1}\right.$ with, typically, $\Delta \omega^{2} \ll \omega_{0}^{2}$ and $\left.\beta_{b}^{2} \ll 1\right)$. In the following, the space charge potential driven by the beating of the two colliding pulses $\phi_{b}\left(\psi_{b}\right)$ will be neglected since $\phi_{b}$ is typically much smaller than the ponderomotive beat wave potential $\left(\hat{a}_{0} \hat{a}_{1}\right)$, as discussed above. Also, since $k_{0}-k_{1} \simeq 2 k_{0}$ (i.e., the width of the beat wave separatrix is approximately $\lambda_{0} / 2$ ), the spatial variation in the pulse envelopes $\hat{a}_{0,1}$, which are assumed to have pulse lengths much greater than $\lambda_{0}$, will be neglected.

The normalized axial momentum of an electron in the beat wave is

$$
u_{z_{b}}\left(\psi_{b}\right)=\beta_{b} \gamma_{b}^{2} H_{b c} \pm \gamma_{b} \sqrt{\gamma_{b}^{2} H_{b c}^{2}-\gamma_{\perp b}^{2}},
$$

where $H_{b c}$ is a constant specifying a given orbit. The $\mathrm{X}$-points are given by $\psi_{\mathrm{x}}=0$ modulo $2 \pi$ and the separatrix is specified by $H_{b}\left(\gamma_{\perp} \gamma_{b} \beta_{b}, 0\right)=\gamma_{\perp}(0) / \gamma_{b}$. The maximum and minimum normalized axial momenta of an electron on a trapped beat wave orbit (extrema of the separatrix) are

$$
u_{b \pm}=\gamma_{b} \beta_{b} \gamma_{\perp b}(0) \pm 2 \gamma_{b} \sqrt{\hat{a}_{0} \hat{a}_{1}}
$$

\section{Trapping threshold}

An approximate threshold for injection into the wakefield can be estimated by applying a phase space separatrix overlap condition (i.e., Chirikov island overlap criterion) [19], assuming circular polarization. Specifically, island overlap requires (i) the maximum momentum of the beat wave separatrix exceed the minimum momentum of the wakefield separatrix and (ii) the minimum momentum of the beat wave separatrix be less than the plasma electron fluid momentum, i.e.,

$$
\begin{aligned}
& u_{b+} \geq u_{z}\left(H=H_{f}\right), \\
& u_{b-} \leq u_{z}(H=1) .
\end{aligned}
$$


If this occurs, then there exists a phase space path that can take an electron from the cold fluid orbit, through the beat wave separatrix, and finally on a trapped orbit within the $2 \mathrm{D}$ separatrix of the wakefield.

The trapping threshold can be solved analytically. In the limit $\beta_{b} \ll 1$, Eqs. (22) and (23) imply

$$
2 \hat{a}_{0} \hat{a}_{1} \simeq \begin{cases}u_{z}\left(H_{f}\right)\left[u_{z}\left(H_{f}\right) / 2-\beta_{b} \sqrt{1+\hat{a}_{0}^{2}+u_{z}^{2}\left(H_{f}\right) / 2}\right], & \text { if } \beta_{b} \leq \beta_{*} \\ u_{z}(H=1)\left[u_{z}(H=1) / 2-\beta_{b} \sqrt{1+\hat{a}_{0}^{2}+u_{z}^{2}(H=1) / 2}\right], & \text { if } \beta_{b}>\beta_{*}\end{cases}
$$

where

$$
\beta^{*}=\frac{\left[u_{z}^{2}\left(H_{f}\right)-u_{z}^{2}(H=1)\right] / \sqrt{2}}{\left[u_{z}\left(H_{f}\right) \sqrt{2+2 \hat{a}_{0}^{2}+u_{z}^{2}\left(H_{f}\right)}-u_{z}(H=1) \sqrt{2+2 \hat{a}_{0}^{2}+u_{z}^{2}(H=1)}\right]} .
$$

Numerical solutions to the analytical estimation of the trapping threshold, Eqs. (22) and (23), are shown in Fig. 2. Note that, in Eqs. (22) and (23), $\hat{a}_{0}$ and $\phi$ are functions of $\psi$, i.e., the relative position within the pump laser pulse. The minimum value of $a_{1}$ required for trapping is plotted versus $a_{0}$ in Fig. 2(a) for different lengths of the drive pulse for $\beta_{b}=0.05$ and $\psi=\psi_{\text {opt }}=-3 \pi / 2$ (i.e., near the back of the pump pulse). Trapping is easiest (occurs for the lowest value of $a_{1}$ for a given $a_{0}$ ) when $L=\lambda_{p}$, which is the resonant case for wakefield generation that yields the largest wakefield amplitude (e.g., $\phi_{0} \simeq 0.4$ for $L=\lambda_{p}$ and $\left.\left\langle a_{0}^{2}\right\rangle=0.5\right)$. Similarly, Fig. 2(b) shows the value of $a_{1}$ required for trapping as a function of the beat wave phase velocity $\beta_{b}$ for several values of $a_{0}$ with $L_{0}=\lambda_{p}$ and $\psi=\psi_{\mathrm{opt}}=-3 \pi / 2$. For these parameters, trapping is optimized for small positive values of $\beta_{p}$. Figure 3 shows an example of the phase space orbits (the $2 \mathrm{D}$ separatrix, the beat wave separatrix, and the fluid orbit) for a case where the island overlap condition is well satisfied $\left(\left\langle a_{0}^{2}\right\rangle=0.5,\left\langle a_{1}^{2}\right\rangle=0.125, \beta_{b}=0.1\right.$ and $\left.L_{0}=\lambda_{p}\right)$.

In the following section, the results of test particle simulations are presented for the linear polarized laser fields discussed in Sec. II. For linear polarization, an analytic theory of the trapping threshold is complicated by the fact that $a^{2}$ is no longer independent of the fast laser phases, i.e., $a^{2}=\hat{a}_{0}^{2} \cos ^{2} \psi_{0}+\hat{a}_{1}^{2} \cos ^{2} \psi_{1}+\hat{a}_{0} \hat{a}_{1}\left[\cos \psi_{b}+\cos \left(\psi_{0}+\psi_{1}\right)\right]$. One consequence is that the wake separatrix now contains fine scale structure since the quantity $\gamma_{\perp 0}^{2}=1+$ $\hat{a}_{0}^{2} \cos ^{2} \psi_{0}$ oscillates between $1+a_{0}^{2}$ and unity. Similarly, the beat wave separatrix becomes "fuzzy" because of contributions from wave components with phases $\cos 2 \psi_{0}, \cos 2 \psi_{1}$, and $\cos \left(\psi_{0}+\psi_{1}\right)$. Furthermore, simulations of the motion of test particles in the beat wave from 
two counterpropagating, linear polarized laser pulses indicates that the particle orbits can become chaotic [20], as discussed in Appendix B. The result is that the trapping threshold is lower than that predicted by circular polarization theory [7], as is apparent in the simulations discussed below.

\section{SIMULATION RESULTS}

This section describes results from a 3D particle tracking code in which the electromagnetic fields for the laser pulses and their corresponding wakefields are specified analytically as described in Sec. II.

In the following simulations, the plasma was modelled by a group of test electrons initially at rest and loaded randomly in a three dimensional spatial region of length $\lambda_{p}$ and transverse size $\lambda_{p} \times \lambda_{p}$, uniformly about the $z$-axis. This spatial region was chosen to be ahead of the pump laser pulse, and timed with respect to the initial position of the injection pulse such that when the two pulses collide, the test electrons fill the entire region in which trapping may occur. After the collision, various properties of the trapped electron bunch were monitored as function of propagation time, such as the mean energy, the energy spread, the root-mean square (rms) bunch length, and the trapping fraction. Here, the trapping fraction is defined as $N_{b} / N_{s}$ where $N_{b}$ is the number of test electrons in the bunch and $N_{s}$ the total number of test electrons in the simulation. Unless otherwise noted, the simulations were carried out for the following parameters ranges: $\left\langle a_{0}^{2}\right\rangle$ from 0.5 to $0.88,\left\langle a_{1}^{2}\right\rangle$ from 0 to 0.32 , drive pulse length from $L_{0}=\lambda_{p}$ to $9 \lambda_{p} / 8$, injection pulse length $L_{1}=\lambda_{p} / 2$, drive and injection pulse radii $r_{i}=\lambda_{p}$, frequencies $\omega_{0}=\omega_{1}=50 \omega_{p}$ and propagation length $\omega_{p} t$ from 50 to 100 . The trapping fraction can be related to the number of trapped particles by $N_{e}=n_{0} f_{\text {tr }} V_{\text {load }}$, where $V_{\text {load }}=\lambda_{p}^{3}$ is the initial volume of loaded test particles.

Three configurations of the two-pulse colliding pulse injector were simulated: (i) two counterpropagating, collinear laser pulses with equal polarizations, (ii) two pulses colliding at a finite interaction angle with equal polarizations, and (iii) two counterpropagating, collinear laser pulses with orthogonal polarizations. 


\section{A. Two collinear pulses}

This section presents results for the basic two-pulse colliding injector geometry in which the pulses are collinear and counterpropagating with equal polarizations. Figure 4 shows the trapped fraction $f_{\operatorname{tr}}$ of electrons, relative energy spread $\Delta \gamma / \gamma, \operatorname{rms}$ bunch length $\sigma_{z} / \lambda_{p}$, rms radius $r_{b} / \lambda_{p}$, and normalized transverse rms emittance $\epsilon_{\perp} / \lambda_{p}$ versus counterpropagating laser pulse intensity after a propagation time of $\omega_{p} t=50$ for the parameters: $\left\langle a_{0}^{2}\right\rangle=0.5$, $\omega_{0} / \omega_{p}=50, L_{0}=9 \lambda_{p} / 8, \omega_{1} / \omega_{p}=50$, and $L_{1}=\lambda_{p} / 2$.

Simulations using the 3D particle tracking code point out that typical electron bunches produced by colliding laser pulses have a "head-to-tail" energy correlation as can be seen in Fig. 6(a), which shows the normalized longitudinal momentum $u_{z}$ versus longitudinal phase $\psi$ for the parameters $\left\langle a_{0}^{2}\right\rangle=0.5, \omega_{0} / \omega_{p}=50, L_{0}=9 \lambda_{p} / 8,\left\langle a_{1}^{2}\right\rangle=0.18, \omega_{1} / \omega_{p}=50$, $L_{1}=\lambda_{p} / 2$, and $\omega_{p} t=100$. The corresponding field profiles versus $\psi$ are shown in Fig. 6(b). In Fig. 6(c), the normalized transverse radial position of the particles $k_{p} r$ is shown versus normalized longitudinal momentum $u_{z}$ for the parameters of Fig. 6(a). The mean kinetic energy of the electron bunch is found to be $T \simeq 17.3 \mathrm{MeV}$. The most energetic electrons that reside at the head of the bunch are on trapped orbits that are both accelerating and focusing and, hence, remain close to the axis. Moving back through the bunch, the electrons are on orbits with less acceleration and less focusing. The least energetic electrons at the back of the bunch reside on orbits that are transversely defocusing and are hence scattered transversely.

The bunch emittance is approximated as $\epsilon_{\perp}=\gamma_{0} \beta_{0} \sqrt{\left\langle x^{2}\right\rangle\left\langle x^{\prime 2}\right\rangle} \simeq \sqrt{\left\langle x^{2}\right\rangle\left\langle u_{x}^{2}\right\rangle}$ where $u_{0}=\gamma_{0} \beta_{0} \simeq \gamma_{0}$ is the axial momentum of the electron bunch. As $a_{1}$ increases, trapping becomes more efficient, with corresponding increases in $f_{\mathrm{tr}}, \Delta \gamma / \gamma, \sigma_{z} / \lambda_{p}$, and $r_{b} / \lambda_{p}$. This is consistent with the fact that the overlap in phase space area between beat wave and wakefield separatrices increases as $a_{1}$ increases, as shown in Fig. 3. Maximum acceptance for the electron plasma wave is obtained for $\left\langle a_{1}^{2}\right\rangle \simeq 0.125$ and, consequently, emittance reaches an asymptotic value. Figure 5 shows the change in trapping fraction $f_{\operatorname{tr}}$ as a function of the beat wave phase velocity for the parameters of Fig. 4 and for the case $\left\langle a_{0}^{2}\right\rangle=0.88$. The maximum of $f_{\operatorname{tr}}$ occurs near $\beta_{b} \simeq 0.35$ for $\left\langle a_{0}^{2}\right\rangle=0.5$ and $\beta_{b} \simeq 0.1$ for $\left\langle a_{0}^{2}\right\rangle=0.88$, which is qualitatively similar to theoretical predictions found for circular polarization (cf. Fig. 2).

The total charge in the bunch $Q$ can be estimated from the trapping fraction $f_{\operatorname{tr}}$ (the 
fraction of the initial electrons that remain on trapped and focused orbits) by $Q=e n_{0} f_{\operatorname{tr}} \lambda_{p}^{3}$. A plot of bunch charge $Q$ versus $a_{1}$ is shown in Fig. 7 for the parameters: $\lambda_{0}=0.8 \mu \mathrm{m}$, $\lambda_{p}=40 \mu \mathrm{m}\left(n_{0}=6.910^{17} \mathrm{~cm}^{-3}\right), L_{0}=r_{0}=40 \mu \mathrm{m}$, and $\left\langle a_{0}^{2}\right\rangle=0.88$. Due to the small volume of the trapped bunch $V_{\mathrm{tr}}$, the bunch density $n_{b}$ can be very high, where $n_{b}=Q / V_{\mathrm{tr}}$. For example, the colliding laser intensities $\left\langle a_{0}^{2}\right\rangle=0.88$ and $\left\langle a_{1}^{2}\right\rangle=0.18$ yield an electron bunch with $r_{b} / \lambda_{p} \simeq 0.1, \sigma_{z} / \lambda_{p} \simeq 0.02, Q \simeq 0.35 \mathrm{nC}$, and $n_{b} / n_{0} \simeq 20$.

Beam loading is important when the trapped electron bunch significantly alters the plasma wave that accelerates the bunch. Beam loading is neglected in the particle tracking code. To estimate the effects of beam loading, the wakefield generated by a short electron bunch in a uniform plasma can be calculated [21, 22] (see Appendix for details). For a uniform beam profile $n_{b}(r, \zeta)=n_{b} \Theta\left(r_{b}-r\right) \Theta(-\zeta) \Theta\left(\zeta+\sigma_{z}\right)$ of radius $r_{b}$ and length $\sigma_{z}$, where $\Theta$ is a step function, the amplitude of the perturbed density and the axial electric field of the bunch-induced wakefield are given by

$$
\begin{aligned}
& \delta n / n_{0} \simeq k_{p} \sigma_{z} n_{b} / n_{0}, \\
& E_{z} / E_{0} \simeq k_{p} \sigma_{z} F_{R}(r) n_{b} / n_{0},
\end{aligned}
$$

assuming $k_{p} \sigma_{z} \ll 1, \delta n / n_{0} \ll 1$, and $E_{z} / E_{0} \ll 1$, where the radial profile function is $F_{R}(r)=1-k_{p} r_{b} K_{1}\left(k_{p} r_{b}\right) I_{0}\left(k_{p} r\right)$ for $r<r_{b}$. Here $I_{0}$ and $K_{1}$ are modified bessel functions and $E_{0}=m_{e} c^{2} k_{p} / e$. For a narrow beam $k_{p}^{2} r_{b}^{2} \ll 1$ and along the axis $F_{R}(r=0) \simeq$ $\left[0.308-0.5 \ln \left(k_{p} r_{b}\right)\right] k_{p}^{2} r_{b}^{2}$. The bunch charge $Q$, normalized bunch-induced axial electric field $E_{z} / E_{0}$, and normalized bunch-induced density perturbation $\delta n / n_{0}$ are plotted in Fig. 8 as a function of $a_{1}$ for $\left\langle a_{0}^{2}\right\rangle=0.88$ and the parameters of Fig. 7. In the regime $\left\langle a_{1}^{2}\right\rangle \gtrsim 0.02$, the density perturbation becomes large, $k_{p} \sigma_{z}\left(n_{b} / n_{0}\right)>1$, and the effects of nonlinear beam loading can no longer be neglected. Nonlinear beam loading will most likely reduce the bunch quality (fraction trapped, average energy, etc.).

The effects of beam loading will be small provided the beam-induced wakefield Eq. (27), is much less than that produced by the drive laser pulse Eq. (12), or

$$
\alpha_{l}=\frac{k_{p} \sigma_{z}}{a_{0}^{2}} \frac{n_{b}}{n_{0}} F_{R}(0) \ll 1
$$

To reduce beam loading, the pump laser amplitude and, consequently, the plasma wave amplitude can be reduced, which also reduces the trapping. For example, $\left\langle a_{0}^{2}\right\rangle=0.5$, $\left\langle a_{1}^{2}\right\rangle=0.18, k_{p} r_{b} \simeq 0.4$, and $k_{p} \sigma_{z} \simeq 0.04$ give a trapped bunch density of $n_{b} / n_{0} \simeq 3.9$ and, 
hence, $\alpha_{l} \simeq 0.03$, which satisfies Eq. (28). The bunch charge $Q$, normalized bunch-induced axial electric field $E_{z} / E_{0}$, and normalized bunch-induced density perturbation $\delta n / n_{0}$ are plotted in Fig. 9 as a function of $a_{1}$ for $\left\langle a_{0}^{2}\right\rangle=0.5$ and the parameters of Fig. 8. For the cases shown in Fig. 9, the effects of beam loading should be minimal.

\section{B. Effects of interaction angle and polarization}

\section{Non-collinear geometry}

Experimentally, the colliding pulse injector geometry can be simplified by using two pulses that intersect in a non-collinear geometry, since this avoids having additional optics in the path of the accelerated electron bunches. The interaction angle $\theta$ between the two laser pulses is given by $\cos \theta=\left(\boldsymbol{k}_{\mathbf{0}} \cdot \boldsymbol{k}_{\mathbf{1}}\right) /\left(k_{0} k_{1}\right)$, where $k_{0}$ propagates along the $z$-axis and $k_{1}$ is in the $x-z$ plane $(\theta=\pi$ corresponds to collinear, counterpropagating pulses). Note for non-collinear interactions, the component of the beat wave phase velocity along the $z$ axis is reduced, i.e., $c \beta_{b_{z}}=\Delta \omega /\left(k_{0}-\left|k_{1}\right| \cos \theta\right), c \beta_{b_{x}}=\Delta \omega /\left(\left|k_{1}\right| \sin \theta\right)$, and $c \beta_{b_{y}}=0$, for $\theta \in(\pi / 2, \pi)$. In addition to the axial (z-axis) component of the beat wave ponderomotive force, proportional to $\left(k_{0}-\left|k_{1}\right| \cos \theta\right) a_{0} a_{1}$, there is now a transverse component, proportional to $\left(\left|k_{1}\right| \sin \theta\right) a_{0} a_{1}$, that pushes electrons off axis. Figure 10 shows the trapping fraction versus interaction angle at $\omega_{p} t=50$ for $\left\langle a_{0}^{2}\right\rangle=0.5, \omega_{0} / \omega_{p}=50, L_{0}=9 \lambda_{p} / 8,\left\langle a_{1}^{2}\right\rangle=0.125$, $\omega_{1} / \omega_{p}=50$, and $L_{1}=\lambda_{p} / 2$. As the angle $\theta$ decreases from $\theta=\pi$ to $\theta=\pi / 2$ (transverse injection), the trapping fraction decreases to zero. Furthermore, for the $\theta=\pi / 2$ case, when the delay between the injection and the pump pulses was increased, such that the injection pulse intersects the wakefield and does not overlap with the pump pulse (as in Umstadter et al. [2], and Hemker et al. [4]), no trapping was observed.

\section{Orthogonal polarization}

All of the above examples have assumed parallel polarization, i.e., $\boldsymbol{a}_{\mathbf{0}} \cdot \boldsymbol{a}_{\mathbf{1}}=a_{0} a_{1}$, and injection is the result of the ponderomotive force associated with the beat wave $\boldsymbol{F}_{\text {beat }}=-\left(m_{e} c^{2} / \gamma\right) \nabla\left(\boldsymbol{a}_{\mathbf{0}} \cdot \boldsymbol{a}_{\mathbf{1}}\right)$. For orthogonal polarizations, $\boldsymbol{F}_{\text {beat }}=0\left(\right.$ since $\left.\boldsymbol{a}_{\mathbf{0}} \cdot \boldsymbol{a}_{\mathbf{1}}=0\right)$, and the beat wave mechanism can no longer be responsible for electron injection. For

orthogonal polarizations, the time-average force on the electrons is given by $\boldsymbol{F}_{\text {pond }}=$ 
$m_{e} c^{2} \nabla(\phi-\gamma) \simeq m_{e} c^{2} \nabla \phi-\left(m_{e} c^{2} / \gamma\right)\left(\nabla \hat{a}_{0}^{2} / 2+\nabla \hat{a}_{1}^{2} / 2\right)$. Electron injection can still be the result of the ponderomotive force associated with envelope of the injection laser pulse, $\boldsymbol{F}_{\mathrm{env}} \simeq-\left(m_{e} c^{2} / \gamma\right) \nabla \hat{a}_{1}^{2} / 2$, but this is relatively small compared to that of the beat wave as discussed in the introduction. As an example, a case was simulated identical to that shown in Fig. 6, except with orthogonal polarization. For the orthogonal polarization case, there are no trapped electrons, compared to $f_{\text {tr }} \simeq 6.5 \times 10^{-4}$ for the parallel polarization case. For orthogonal polarization, trapping can occur, but for higher laser intensities in which nonlinear effects (not included in the test particle simulation model) become important. The details of trapping using orthogonal polarizations are presently being explored using particle-in-cell simulations [23].

\section{CONCLUSION}

An alternative configuration of the colliding pulse injector that uses a single pump pulse and a single counterpropagating injection pulse has been analyzed and simulated. This single injection pulse configuration has the advantages of simplicity and ease of experimental implementation. Injection is the result of the slow ponderomotive beat wave generated when the backward injection pulse collides with the rear portion of the forward pump pulse. Injection requires high pump laser intensity $\left(a_{0} \simeq 1\right)$ and modest injection pulse intensity $\left(a_{1} \simeq 0.2\right)$. Test particle simulations indicate that significant amounts of charge can be trapped and accelerated $(Q \sim 10 \mathrm{pC})$, up to the limits imposed by beam loading. In addition, the accelerated bunches are ultrashort $(\sim 1 \mathrm{fs})$ with good beam quality $(\Delta \gamma / \gamma \sim$ few percent at a mean energy of $\sim 10 \mathrm{MeV}$ and a normalized rms emittance on the order $0.4 \mathrm{~mm} . \mathrm{mrad}$ ). Reduction of the energy spread can be achieved by including a density taper in the trapping region. The density taper will rephase electrons and consequently reduce the energy spread and increase the bunch charge. Also examined was the effect of interaction angle. For an interaction angle of $150^{\circ}$ (where $180^{\circ}$ is collinear, counterpropagating), the trapping fraction is only reduced by roughly ten percent, thus allowing efficient non-collinear interaction geometries for further ease of experimental implementation. When the interaction angle was decreased to $90^{\circ}$ (transverse injection geometry), no trapping was observed for the parameters of the simulation. Similarly, no trapping was observed for the parameters of the simulations for the case of orthogonal polarization. This confirms that the mechanism 
responsible for injection is the result of the slow ponderomotive force associated with the beating of the laser pulses, and not due to ponderomotive force associated with the envelope of the injection pulse.

One limitation of the approach used in this research is that it relies on test particle simulations in which the fields (lasers and wakes) were specified analytically. This model becomes inaccurate as $a_{0}$ and $a_{1}$ approach and exceed unity, since analytical expressions for nonlinear wakefields in 3D are lacking in the literature. Self-consistent simulations, such as using particle-in-cell codes, are required in this nonlinear regime, and this line of research is currently being pursued. Likewise, experiments on colliding pulse injection are being pursued at LBNL, as well as other laboratories world wide.

\section{ACKNOWLEDGMENTS}

The authors acknowledge useful discussions with J. Faure, B. A. Shadwick, J. L. Bobin, J. R. Cary and R. Giacone. This work was performed under the auspices the U.S. Department of Energy, Office of High Energy Physics, under contract number DE-AC-03-76SF0098, and by the U.S. Department of Energy SciDAC project, "Advanced Computing for 21st Century Accelerator Science and Technology", which is supported by the Office of High Energy Physics and the Office of Advanced Scientific Computing Research.

\section{APPENDIX A: BEAM LOADING CONSIDERATIONS}

Beam loading, whereby the trapped electron bunch significantly alters the accelerating wakefield, can degrade the quality of the electron bunch. To estimate the effects of beam loading, the wakefield generated by the trapped electron bunch propagating in an initially uniform plasma can be calculated [21, 22] and compared to the wakefield driven by the pump laser pulse. Using linear perturbation theory of the cold fluid-Maxwell equations (i.e., to

first order in $\delta n / n_{0}, E_{z} / E_{0}, a^{2}$, etc.), the normalized density perturbation $\delta n / n_{0} \ll 1$ and normalized axial electric field $E_{z} / E_{0} \ll 1$ driven in an initially uniform plasma by either a 
short electron bunch $\left(n_{b} / n_{0}\right.$ drive term) or a short laser pulse ( $a^{2}$ drive term) are given by

$$
\begin{aligned}
\left(\frac{\partial^{2}}{\partial \zeta^{2}}+k_{p}^{2}\right) \frac{\delta n}{n_{0}} & =\left(\nabla_{\perp}^{2}+\frac{\partial^{2}}{\partial \zeta^{2}}\right) \frac{\left\langle a^{2}\right\rangle}{2}-k_{p}^{2} \frac{n_{b}}{n_{0}}, \\
\left(\nabla_{\perp}^{2}-k_{p}^{2}\right) \frac{E_{z}}{E_{0}} & =k_{p} \frac{\partial}{\partial \zeta}\left(\frac{\left\langle a^{2}\right\rangle}{2}-\frac{\delta n}{n_{0}}\right),
\end{aligned}
$$

where $n_{b}$ is the density of the drive electron bunch, $n_{0}$ is the ambient plasma density, $E_{0}=k_{p} m_{e} c^{2} / e$ the cold fluid wave breaking limit, $v_{b} \simeq c$ was assumed $\left(v_{b}\right.$ is the bunch velocity), and the angular brackets denote an average over the fast laser frequency (with $\left.\omega^{2} \gg \omega_{p}^{2}\right)$. In deriving the above equations, the quasi-static approximation was assumed, i.e, the drive beams and the resulting wakefields are functions of only the variables $\zeta=z-c t$ and $r_{\perp}$.

Consider the wakefield generated by the electron bunch in the absence of the laser pulse. Solving Eq. (A2) with $a^{2}=0$ and a cylindrically-symmetric drive $n_{b}$ yields

$$
\begin{aligned}
& \frac{\delta n}{n_{0}}=k_{p} \int_{0}^{\zeta} d \zeta^{\prime} \sin \left[k_{p}\left(\zeta-\zeta^{\prime}\right)\right] \frac{n_{b}\left(\zeta^{\prime}\right)}{n_{0}}, \\
& \frac{E_{z}}{E_{0}}=k_{p}^{3} \int_{\infty}^{\zeta} d \zeta^{\prime} \int_{0}^{\infty} d r^{\prime} r^{\prime} \cos \left[k_{p}\left(\zeta-\zeta^{\prime}\right)\right] I_{0}\left(k_{p} r_{<}\right) K_{0}\left(k_{p} r_{>}\right) \frac{n_{b}\left(r^{\prime}, \zeta^{\prime}\right)}{n_{0}},
\end{aligned}
$$

where $I_{0}$ and $K_{0}$ are the zeroth-order modified bessel functions of the second kind, and $r_{<}\left(r_{>}\right)$denote the smaller (larger) of $r$ and $r^{\prime}$ respectively. For a uniform beam profile $n_{b}(r, \zeta)=n_{b} \Theta\left(r_{b}-r\right) \Theta(-\zeta) \Theta\left(\zeta+\sigma_{z}\right)$ of radius $r_{b}$ and length $\sigma_{z}$, where $\Theta$ is a step function, the amplitudes of the perturbed density and the axial wakefield are

$$
\begin{aligned}
& \frac{\delta n}{n_{0}} \simeq k_{p} \sigma_{z} \frac{n_{b}}{n_{0}}, \\
& \frac{E_{z}}{E_{0}} \simeq k_{p} \sigma_{z} \frac{n_{b}}{n_{0}} F_{R}(r),
\end{aligned}
$$

assuming $\sigma_{z} \ll 1$, where the radial profile function is

$$
F_{R}(r)= \begin{cases}1-k_{p} r_{b} K_{1}\left(k_{p} r_{b}\right) I_{0}\left(k_{p} r\right), & \text { for } r<r_{b} \\ k_{p} r_{b} I_{1}\left(k_{p} r_{b}\right) K_{0}\left(k_{p} r\right), & \text { for } r>r_{b}\end{cases}
$$

with $I_{1}$ and $K_{1}$ the first-order modified bessel functions.

Consider the wakefield generated by the laser pulse in the absence of the electron bunch. Solving Eq. (A2) with $n_{b}=0$ yields

$$
\begin{aligned}
& \frac{\delta n}{n_{0}}=k_{p} \int_{0}^{\zeta} d \zeta^{\prime} \sin \left[k_{p}\left(\zeta-\zeta^{\prime}\right)\right]\left(\nabla_{\perp}^{2}+\frac{\partial^{2}}{\partial \zeta^{\prime 2}}\right) \frac{\left\langle a^{2}\left(\zeta^{\prime}\right)\right\rangle}{2}, \\
& \frac{E_{z}}{E_{0}}=k_{p} \int_{0}^{\zeta} d \zeta^{\prime} \sin \left[k_{p}\left(\zeta-\zeta^{\prime}\right)\right] \frac{\partial}{\partial \zeta^{\prime}} \frac{\left\langle a^{2}\left(\zeta^{\prime}\right)\right\rangle}{2} .
\end{aligned}
$$


Assuming a laser pulse with a half-sine axial profile and a Gaussian radial profile, similar to Eq. (5), with a pulse length $L=\lambda_{p}$ (the resonant case yielding maximum plasma wave amplitude) gives

$$
\begin{aligned}
& \frac{\delta n}{n_{0}}=\frac{\pi}{8} a_{0}^{2}\left[1+\frac{8}{k_{p}^{2} r_{s}^{2}}\left(1-\frac{2 r^{2}}{r_{s}^{2}}\right)\right] \exp \left(-\frac{2 r^{2}}{r_{s}^{2}}\right) \\
& \frac{E_{z}}{E_{0}}=\frac{\pi}{8} a_{0}^{2} \exp \left(-\frac{2 r^{2}}{r_{s}^{2}}\right)
\end{aligned}
$$

Beam loading can be neglected provided that the wakefield generated by the trapped electron bunch is small compared to that generated by the drive laser pulse. Consequently, from Eqs. (A6) and (A11), beam loading can be neglected provided

$$
\alpha_{l}=\frac{k_{p} \sigma_{z}}{a_{0}^{2}} \frac{n_{b}}{n_{0}} F_{R}(0) \ll 1 .
$$

For the parameters under consideration in this paper (e.g., $\left\langle a_{0}^{2}\right\rangle=0.5,\left\langle a_{0}^{2}\right\rangle=0.18, k_{p} r_{b} \simeq$ $0.4, k_{p} \sigma_{z} \simeq 0.04$, and $n_{b} / n_{0} \simeq 3.9$, which yields $\left.\alpha_{l} \simeq 0.03\right)$, Eq. (A12) is satisfied.

\section{APPENDIX B: EFFECT OF POLARIZATION ON ELECTRON MOTION IN BEAT WAVES}

Test particle simulations of the colliding pulse injection process indicate that trapping occurs more readily for the case of linear polarization compared to that of circular polarization. One reason for this difference is the form of the normalized laser intensity (i.e., the ponderomotive potential) for the two polarizations. Consider the case of two counterpropagating and overlapping laser pulses with uniform profiles (i.e., pulse rise time effects are neglected). For circular polarization $\mathbf{a}=\sum_{i} \hat{a}_{i}\left(\cos \psi_{i} \mathbf{e}_{x}+\sin \psi_{i} \mathbf{e}_{y}\right)$, such that $a^{2}=\hat{a}_{0}^{2}+\hat{a}_{1}^{2}+2 \hat{a}_{0} \hat{a}_{1} \cos \psi_{b}$ is independent of the fast laser phase $\psi_{i}$ and only a function of the beat phase $\psi_{b}=\psi_{0}-\psi_{1} \simeq 2 k_{0} z-\Delta \omega t$. Because $a^{2}=a^{2}\left(\psi_{b}\right)$, the Hamiltonian for the motion of an electron in the combined laser fields is time independent, $H_{b}\left(u_{z}, \psi_{b}\right)=\sqrt{1+a^{2}\left(\psi_{b}\right)+u_{z}^{2}}-\beta_{b} u_{z}$, i.e., the Hamiltonian describes the motion of an electron in a single beat wave characterized by a single phase velocity. In this case the electron motion is regular as describe in Sec. III B.

For linear polarization $\mathbf{a}=\sum_{i} \hat{a}_{i} \cos \psi_{i} \mathbf{e}_{x}$, such that $a^{2}=\hat{a}_{0}^{2} \cos ^{2} \psi_{0}+\hat{a}_{1}^{2} \cos ^{2} \psi_{1}+$ $\hat{a}_{0} \hat{a}_{1}\left[\cos \psi_{b}+\cos \left(\psi_{0}+\psi_{1}\right)\right]$. In this case the ponderomotive potential is, in effect, composed of four waves. In addition to the slow beat wave $\hat{a}_{0} \hat{a}_{1} \cos \psi_{b}$, there is a forward going 
wave $\hat{a}_{0}^{2} \cos ^{2} \psi_{0}$, a backward going wave $\hat{a}_{1}^{2} \cos ^{2} \psi_{1}$, and a wave at the sum of the laser phases $\hat{a}_{0} \hat{a}_{1} \cos \left(\psi_{0}+\psi_{1}\right)$. The end result is that the Hamiltonian is no longer time independent and an analytic solution for the motion of a test electron in the combined laser fields is intractable.

To study the effect of polarization, the motion of test particles is studied numerically for two identical, counterpropagating laser pulses, neglecting the effects of the space charge potential $(\phi=0)$. The first set of simulations, shown in Fig. 11, is for two overlapping pulses with uniform profiles, i.e., the effects of the laser envelope profiles are neglected. In this case, a group of electrons is initially loaded at rest over one-half of a beat period (spaced uniformly) within the laser fields. Here, the time-averaged intensities of the circularly and linearly polarized laser pulses are chosen to be equal, $\left\langle a_{0}^{2}\right\rangle=\left\langle a_{1}^{2}\right\rangle=0.5$.

Results for circular polarization are shown in Fig. 11 (a), which indicates that the orbits are regular and well behaved, as is described by the Hamiltonian theory in Sec. III B. In this case, the maximum electron momentum in the beat wave is less than or equal to that of the maximum of the beat wave separatrix given by Eq. (20).

The corresponding case for linear polarization is shown in Fig. 11 (b). For linear polarization, the orbits are irregular and, for sufficiently intense laser pulses, can become chaotic. This is similar to the case of "stochastic heating" in counterpropagating laser fields as studied by Sheng et al. [20]. In this case the electrons are not confined to a single beat wave period and the maximum momentum can exceed that of the maximum of the beat wave separatrix as predicted by a Hamiltonian theory for circularly polarized pulses.

To study the effect of the finite rise times of the laser pulses, a second set of simulations was performed. In these simulations, the electrons were loaded at rest in the region between the two counterpropagating laser pulses before they overlapped (spaced uniformly over a width equal to half of a beat period). The initial conditions were such that the electrons were first struck by the left-going pulse for a short time (less than a beat period) before being struck by the right-going pulse (at which time the electrons experience the beat wave). Here the laser pulses have a finite length of $L_{0}=L_{1}=50 \lambda_{0}$ and a radius of $r_{0}=r_{1}=50 \lambda_{0}$ with equal peak time-averaged intensities of $\left\langle a_{0}^{2}\right\rangle=\left\langle a_{1}^{2}\right\rangle=0.5$.

The case of circular polarization is shown in Fig. 12 (a). Initially, the electrons move to the left due the axial ponderomotive force of the left-going pulse. As the two pulses collide, the electrons begin to execute orbits within the beat wave. As the laser pulses continue to 
overlap, the size of the ponderomotive beat wave increases, since the local laser intensity of the two pulses is increasing. This leads to larger beat wave orbits. The end result is that the electrons are confined to a single period of the beat wave and, for these initial conditions, the maximum momentum is significantly less than that corresponding to the top of the beat wave separatrix given by Eq. (20).

The case of linear polarization is shown in Fig. 12 (a). Again, the electron orbits are highly irregular and chaotic. The electrons are not confined to a single beat wave period and the maximum momentum exceeds that predicted by a simple Hamiltonian theory of the separatrix for circularly polarized pulses.

The above simulations shed insight as to why injection and trapping occurs more readily for linear polarization than it does for circular polarization. For circular polarization, electrons initially loaded at rest within a single beat wave period remain confined to a single period of the beat wave with momenta less than that of the beat wave separatrix. For linear polarization, the electron trajectories become chaotic, no longer confined to a single beat wave period, and obtain momenta exceeding that predicted by the separatrix corresponding to the circular polarization case. The fact that linear polarization results in large phase excursions as well as large momentum gains, in comparison to circular polarization, implies that the use of linear polarization can be more effective than circular polarization in the beat wave injection and trapping of electrons. 

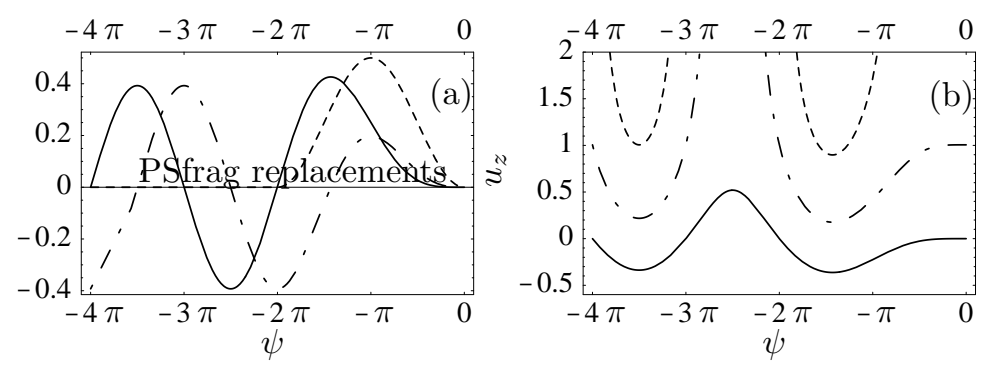

FIG. 1: (a) Wakefield $\phi$ (solid line), drive laser pulse envelope $\left\langle a^{2}\right\rangle$ (dashed line), and longitudinal electric field $E_{z}=-\partial_{z} \phi$ (dot-dashed line) for $L_{0}=\lambda_{p}$ and $\left\langle a_{0}^{2}\right\rangle=0.5$ (b) Phase space plot showing cold fluid orbit (solid line), trapped and focused orbit (dashed line), and trapped separatrix (dotdashed line).
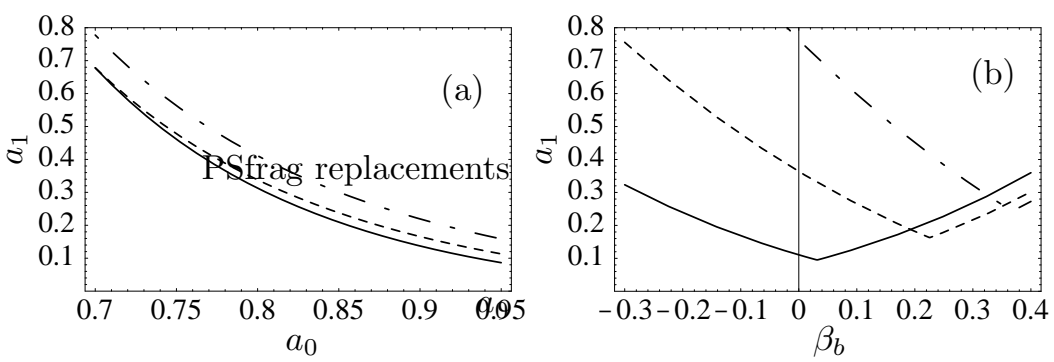

FIG. 2: (a) Injection laser pulse amplitude $a_{1}$ versus pump laser pulse amplitude $a_{0}$ at threshold for $\psi_{\mathrm{opt}}=-3 \pi / 2, \beta_{b} \simeq 0.05, L_{0}=\lambda_{p}$ (solid line), $L_{0}=9 \lambda_{p} / 8$ (dashed line), and $L_{0}=5 \lambda_{p} / 4$ (dot-dashed line). (b) Injection laser pulse amplitude $a_{1}$ versus $\beta_{b}$ at threshold for $\psi_{\text {opt }}=-3 \pi / 2$, $L_{0}=\lambda_{p},\left\langle a_{0}^{2}\right\rangle=0.45$ (solid line), $\left\langle a_{0}^{2}\right\rangle=0.32$ (dashed line), and $\left\langle a_{0}^{2}\right\rangle=0.245$ (dot-dashed line).

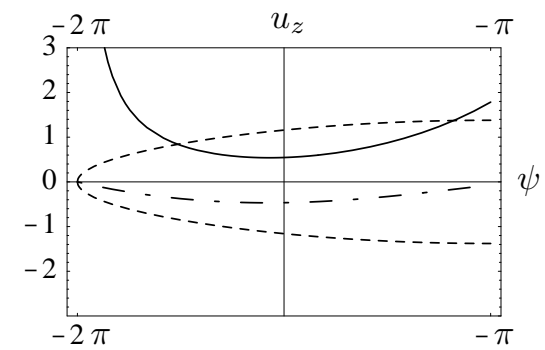

FIG. 3: Phase-space $\left(\psi, u_{z}\right)$ showing trapped and focused separatrix (solid line), cold fluid orbit (dashed line), and maximum of the beat wave separatrix (dot-dashed line) for $\left\langle a_{0}^{2}\right\rangle=0.45,\left\langle a_{1}^{2}\right\rangle=$ $0.125, \beta_{b}=0$, and $L_{0}=\lambda_{p}$. 


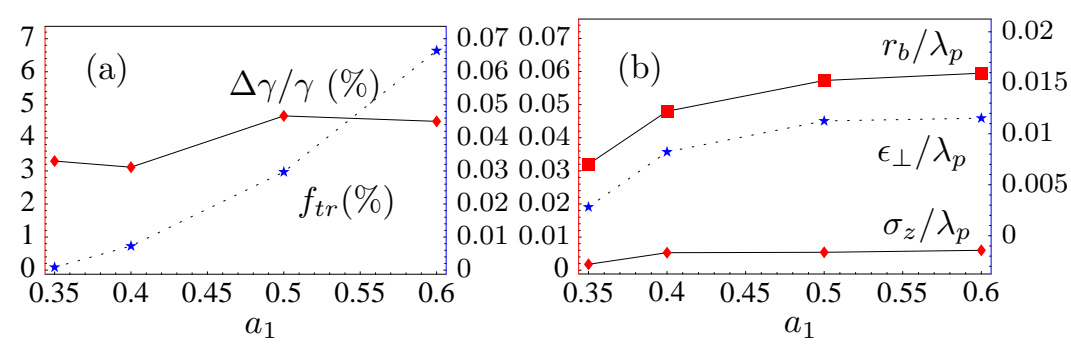

FIG. 4: Trapped bunch parameters versus $a_{1}$ (for two collinear, counterpropagating laser pulses with equal polarization, $\left\langle a_{0}^{2}\right\rangle=0.5, \omega_{0} / \omega_{p}=50, L_{0}=9 \lambda_{p} / 8, \omega_{1} / \omega_{p}=50, L_{1}=\lambda_{p} / 2$, and $\omega_{p} t=50$ ). (a) Trapping fraction $f_{\text {tr }}$ (right vertical axis) and relative energy spread $\Delta \gamma / \gamma$ (left vertical axis). (b) Bunch length $\sigma_{z} / \lambda_{p}$ (left vertical axis), rms radius $r_{b} / \lambda_{p}$ (left vertical axis), and normalized transverse rms emittance $\epsilon_{\perp} / \lambda_{p}$ (right vertical axis).
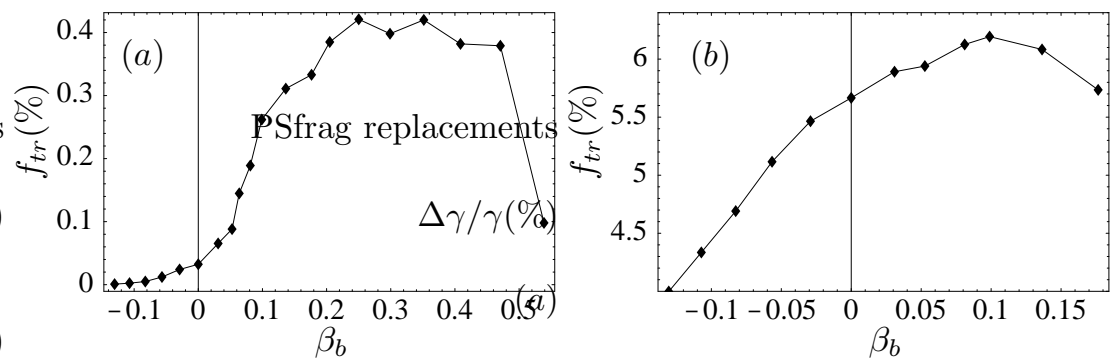

FIG. 5: Trapping fraction $f_{\text {tr }}$ as a function of beat wave phase velocity $\beta_{b}$ for two collinear, counterpropagating laser pulses with equal polarization for the parameters: $(a)\left\langle a_{0}^{2}\right\rangle=0.5, \omega_{0}=$ $50 \omega_{p}, L_{0}=9 \lambda_{p} / 8,\left\langle a_{1}^{2}\right\rangle=0.125, L_{1}=\lambda_{p} / 2, \omega_{p} t=50$ and $(b)$ same parameters except with $\left\langle a_{0}^{2}\right\rangle=0.88$ and $\left\langle a_{1}^{2}\right\rangle=0.245$. 

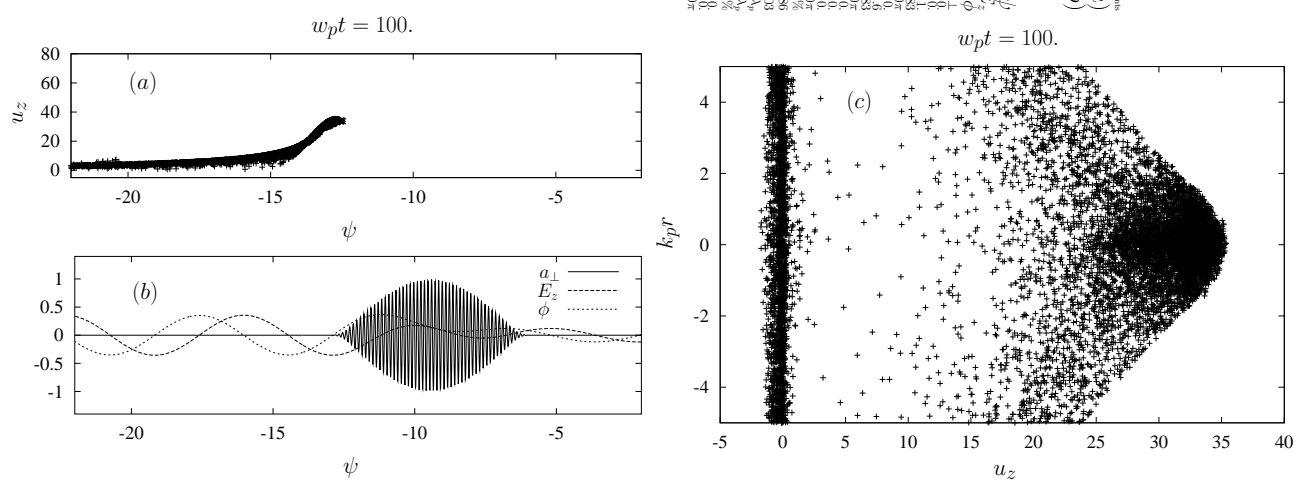

FIG. 6: (a) Longitudinal electron momentum $u_{z}$ versus phase $\psi=k_{p} \zeta$. (b) Normalized laser strength $a_{\perp}$ (solid curve), longitudinal electric field $E_{z}$ (dashed curve) and wakefield potential $\phi$ (dotted curve) versus phase (note that the trapped and focused region is $-4 \pi<\psi<-7 \pi / 2$ ). (c) Longitudinal momentum versus normalized beam radius $k_{p} r$. Laser-plasma parameters: $\left\langle a_{0}^{2}\right\rangle=0.5$, $\omega_{0} / \omega_{p}=50, L_{0}=9 \lambda_{p} / 8,\left\langle a_{1}^{2}\right\rangle=0.18, \omega_{1} / \omega_{p}=50, L_{1}=\lambda_{p} / 2$, parallel polarization, and $\omega_{p} t=100$.

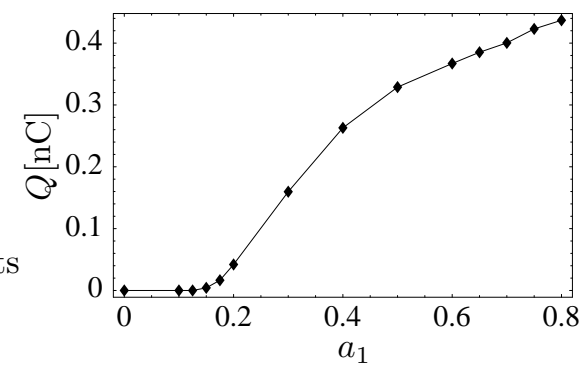

FIG. 7: Bunch charge $Q[\mathrm{nC}]$ versus $a_{1}$ with $\lambda_{0}=0.8 \mu \mathrm{m}, \lambda_{p}=40 \mu \mathrm{m}\left(n_{0}=6.910^{17} \mathrm{~cm}^{-3}\right)$, $L_{0}=r_{0}=40 \mu \mathrm{m}$, and $\left\langle a_{0}^{2}\right\rangle=0.88$.

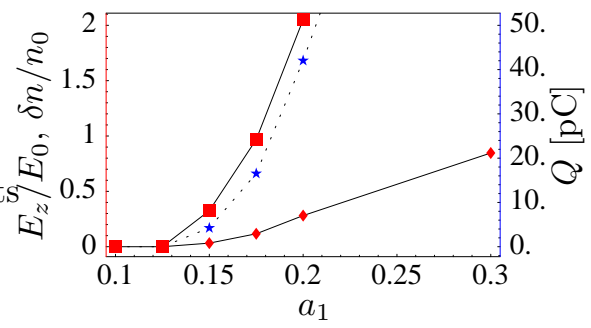

FIG. 8: Bunch charge $Q$ in pC (right vertical axis, stars), normalized axial electric field $E_{z} / E_{0}$ (left vertical axis, points), and normalized density perturbation $\delta n / n_{0}$ (left vertical axis, squares) generated by the electron bunch alone (here the laser contribution is not included) versus $a_{1}$ with $\lambda_{0}=0.8 \mu \mathrm{m}, \lambda_{p}=L_{0}=r_{0}=40 \mu \mathrm{m}$, and $\left\langle a_{0}^{2}\right\rangle=0.88$. 


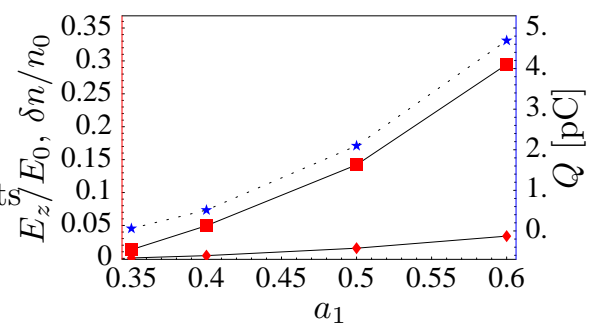

FIG. 9: Bunch charge $Q$ in pC (right vertical axis, stars), normalized axial electric field $\left(E_{z} / E_{0}\right)$ (left vertical axis, points), and normalized density perturbation $\left(\delta n / n_{0}\right)$ (left vertical axis, squares) generated by the electron bunch alone versus $a_{1}$ with $\lambda_{0}=0.8 \mu \mathrm{m}, \lambda_{p}=40 \mu \mathrm{m}$ $\left(n_{0}=6.910^{17} \mathrm{~cm}^{-3}\right), L_{0}=r_{0}=40 \mu \mathrm{m}$, and $\left\langle a_{0}^{2}\right\rangle=0.5$. Note that the parameter regime is well below the beam loading limit.

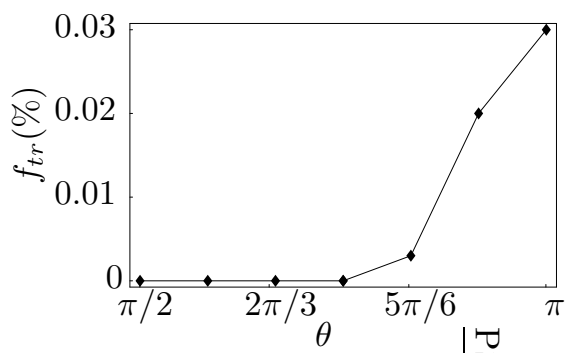

FIG. 10: Trapping fraction $f_{\operatorname{tr}}$ as a function of angle for two laser beams with equal polarization at $\omega_{p} t=50$ with $\left\langle a_{0}^{2}\right\rangle=0.5, \omega_{0} / \omega_{p}=50, L_{0}=9 \lambda_{p} / 8,\left\langle a_{0}^{2}\right\rangle=0.125, \omega_{1} / \omega_{p}=50$, and $L_{1}=\lambda_{p} / 2$.
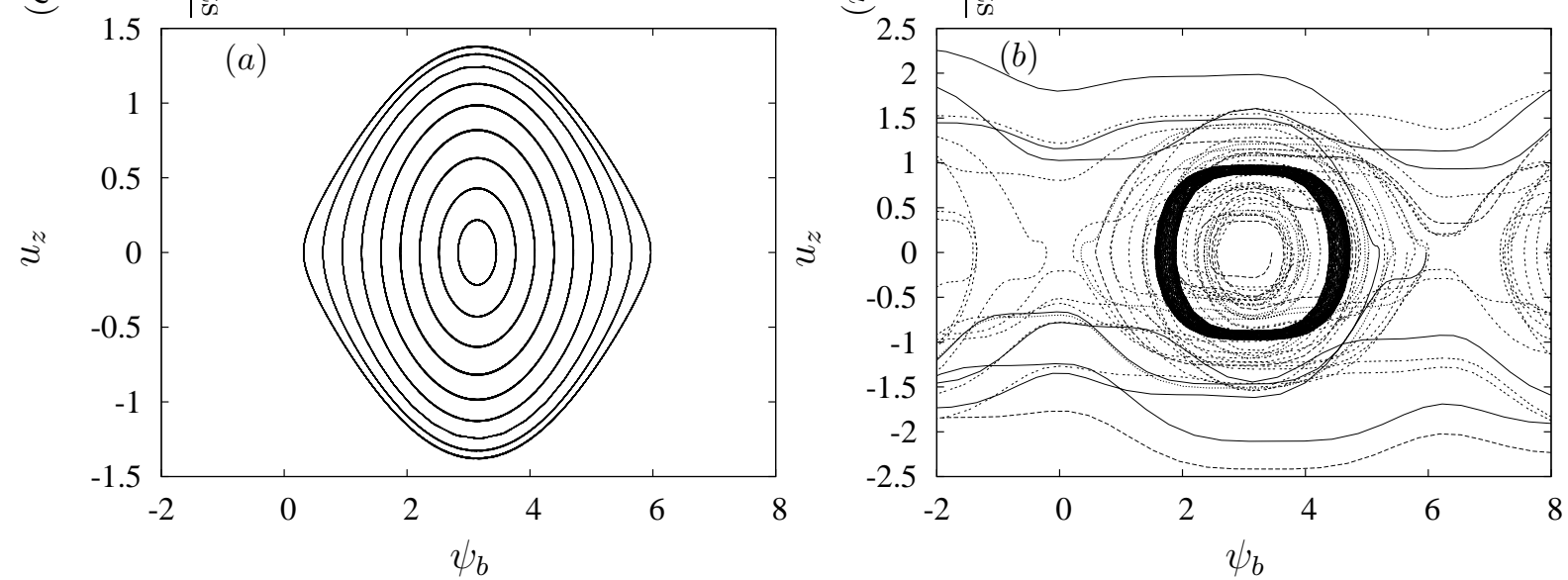

FIG. 11: Phase space orbits $\left(u_{z}, \psi_{b}\right)$ of test electrons in two counterpropagating laser pulses with (a) circular polarization and (b) linear polarization. Here both lasers are infinite plane waves with $\left\langle a_{0}^{2}\right\rangle=\left\langle a_{1}^{2}\right\rangle=0.5$, i.e., equal time-averaged intensities. 

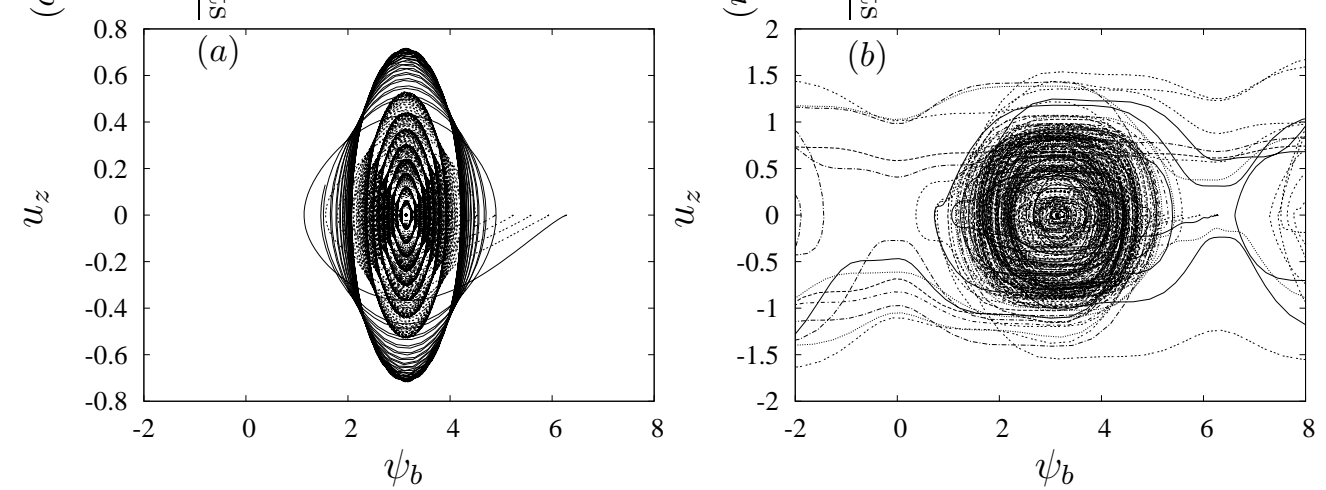

FIG. 12: Phase space orbits $\left(u_{z}, \psi_{b}\right)$ of test electrons in two counterpropagating laser pulses with (a) circular polarization and (b) linear polarization. Here the laser pulses have a finite length of $L_{0}=L_{1}=50 \lambda_{0}$ and a radius of $r_{0}=r_{1}=50 \lambda_{0}$ with equal peak time-averaged intensities of $\left\langle a_{0}^{2}\right\rangle=\left\langle a_{1}^{2}\right\rangle=0.5$. 
[1] E. Esarey, P. Sprangle, J. Krall, and A. Ting, IEEE Trans. Plasma Sci. 24, 252 (1996).

[2] D. Umstadter, J. K. Kim, and E. Dodd, Phys. Rev. Lett. 76, 2073 (1996).

[3] E. Esarey, R. F. Hubbard, W. P. Leemans, A. Ting, and P. Sprangle, Phys. Rev. Lett. 79, 2682 (1997).

[4] R. G. Hemker, K.-C. Tzeng, W. B. Mori, C. E. Clayton, and T. Katsouleas, Phys. Rev. E 57, 5920 (1998).

[5] S. Bulanov, N. Naumova, F. Pegoraro, and J. Sakai, Phys. Rev. E 58, R5257 (1998).

[6] C. B. Schroeder, P. B. Lee, J. S. Wurtele, E. Esarey, and W. P. Leemans, Phys. Rev. E 59, 6037 (1999).

[7] E. Esarey, C. B. Schroeder, W. P. Leemans, and B. Hafizi, Phys. Plasmas 6, 2262 (1999).

[8] J. Krall, E. Esarey, P. Sprangle, and G. Joyce, Phys. Plasmas 1, 1738 (1994).

[9] A. Modena, Z. Najmudin, A. E. Dangor, C. E. Clayton, K. A. Marsh, C. Joshi, V. Malka, C. B. Darrow, C. Danson, D. Neely, et al., Nature 377, 606 (1995).

[10] R. Wagner, S.-Y. Chen, A. Maksimchuk, and D. Umstadter, Phys. Rev. Lett. 78, 3125 (1997).

[11] C. I. Moore, A. Ting, K. Krushelnick, E. Esarey, R. F. Hubbard, B. Hafizi, H. R. Burris, C. Manka, and P. Sprangle, Phys. Rev. Lett. 79, 3909 (1997).

[12] W. P. Leemans, D. Rodgers, P. E. Catravas, C. G. R. Geddes, G. Fubiani, E. Esarey, B. A. Shadwick, R. Donahue, and A. Smith, Phys. Plasmas 8, 2510 (2001).

[13] W. P. Leemans, P. Catravas, E. Esarey, C. G. R. Geddes, C. Toth, R. Trines, C. B. Schroeder, B. A. Shadwick, J. van Tilborg, and J. Faure, Phys. Rev. Lett. 89, 174802 (2002).

[14] V. Malka, S. Fritzler, E. Lefebvre, M.-M. Aleonard, F. Burgy, J.-P. Chambaret, J.-F. Chemin, K. Krushelnick, G. Malka, S. P. D. Mangles, et al., Science 298, 1596 (2002).

[15] D. Gordon, K.-C. Tzeng, C. E. Clayton, A. E. Dangor, V. Malka, K. A. Marsh, A. Modena, W. B. Mori, P. Muggli, Z. Najmudin, et al., Phys. Rev. Lett. 80, 2133 (1998).

[16] E. Esarey, B. Hafizi, R. Hubbard, and A. Ting, Phys. Rev. Lett. 80, 5552 (1998).

[17] E. Esarey, C. B. Schroeder, and W. P. Leemans, in Femtosecond Beam Science, edited by M. Uesaka (World Scientific Press, in press).

[18] E. Esarey and W. P. Leemans, Phys. Rev. E 59, 1082 (1999).

[19] A. J. Lichtenberg and M. A. Lieberman, Regular and Stochastic Motion (Springer-Verlag, New 
York, 1983).

[20] Z.-M. Sheng, K. Mima, Y. Sentoku, M. Jovanović, T. Taguchi, J. Zhang, and J. Meyer-terVehn, Phys. Rev. Lett. 88, 055004 (2002).

[21] R. Keinings and M. E. Jones, Phys. Fluids 30, 252 (1987).

[22] T. Katsouleas, S. Wilks, P. Chen, J. M. Dawson, and J. J. Su, in Part. Accel. Conf. (1987), vol. 22 , pp. 81-99.

[23] J. Cary, R. Giacone, C. Nieter, E. Esarey, G. Fubiani, and W. P. Leemans, in Part. Accel. Conf. (2003), vol. 72, p. 490. 